EXPRESSION PROFILING AND COMPARATIVE SEQUENCE DERIVED INSIGHTS INTO

LIPID METABOLISM

Words: 2931

Matthew J. Callow and Edward M. Rubin

Genome Sciences Department

Lawrence Berkeley National Laboratory

1 Cyclotron Rd

Berkeley CA 94720

Tel: +1-510 4952252

Fax: +1-510 4864229

Email: mjcallow@lbl.gov 


\begin{abstract}
Expression profiling and genomic DNA sequence comparisons are increasingly being applied to the identification and analysis of the genes involved in lipid metabolism. Not only has genome-wide expression profiling aided in the identification of novel genes involved in important processes in lipid metabolism such as sterol efflux, but the utilization of information from these studies has added to our understanding of the regulation of pathways participating in the process. Coupled with these gene expression studies, cross species comparison, searching for sequences conserved through evolution, has proven to be a powerful tool to identify important non-coding regulatory sequences as well as the discovery of novel genes relevant to lipid biology. An example of the value of this approach was the recent chance discovery of a new apolipoprotein gene (apo AV) that has dramatic effects upon triglyceride metabolism in mice and humans.
\end{abstract}


Recent years have seen an explosion in genomic resources including a variety of new strategies to address how genes are regulated. Genome-wide expression profiling has been a popular approach to identify patterns of gene expression that may involve common regulatory mechanisms. The recent abundance of genomic sequence data has also led to a focus on multiorganism sequence comparisons to annotate the human genome including the identification of sequences participating in gene regulation. Comparative sequence based approaches for annotating the human genome are based on the observation that sequences that are conserved between multiple species are likely to be important based on selective pressures to maintain these sequences. This review aims to examine recent developments in gene expression and comparative sequence analysis and how they are advancing our knowledge of the genes and pathways relevant to lipid metabolism.

\section{Microarray technology}

There are currently two common platforms for microarray expression profiling. The first uses high-density oligonucleotide synthesis on a glass surface while the second prints PCRamplified DNA, usually from cloned cDNA products [1, 2]. In addition, the printing of presynthesized oligonucleotides of 50-70 bases in length has also seen increasing popularity. Both technologies rely upon the hybridization of the arrays with a pool of labeled targets, usually representing a complex mixture of mRNAs of a biological sample or a combination of experimental and control samples. Ratios of signal intensity are used to determine the relative abundance of mRNA for the samples being tested compared with a reference sample.

\section{Data analysis and collection}

Once signal intensity data is collected from the arrays it needs to be analyzed in a meaningful way. Some of the common issues that confront the analysis of data from microarray experiments include 1) issues of signal intensity that could be affected by target or probe abundance, 2) background noise, and 3) the random chance of obtaining extreme ratios. The way these issues are dealt with varies widely in the literature, however a common approach is to 
perform arbitrary intensity and ratio cutoffs of multiple experiments to identify genes that are differentially expressed between two groups under study. In a recent study to identify those genes that had altered expression in HDL metabolism, transgenic and knockout mouse liver gene expression was ranked based upon the t-statistic value determined from the gene-modified group and a control group [3*]. This approach avoids the necessity of assigning intensity and ratio cutoffs because of the inherent variability of low intensity genes which tend to decrease t-statistic values. However, no matter what approach is used for identifying differentially expressed genes, it is commonly recognized that alternative follow-up strategies need to be employed to generate confidence in the findings of the array studies. Several recent reviews have addressed the issue of microarray data analysis [4-6].

While performing independent replicated experiments is important in drawing conclusions from microarray experiments and reducing the likelihood of identifying false-positives, single experiments may still be a useful screening strategy depending upon the expected outcomes. This is exemplified in work by Buechler et al $\left[7^{\star}\right]$ where a single array experiment on monocyte mRNA from a patient with high Lipoprotein(a) $(\operatorname{Lp}(a))$ levels and a patient with low $L p(a)$ was performed to identify genes that were differentially expressed. The information from a single array was then used to examine additional patients using alternative, less high-throughput techniques. They found that the mRNA levels for plasminogen activator inhibitor 2 were significantly altered between the high and low $L p(a)$ patients after first identifying this candidate from the microarray screen. In this case differentially expressed genes that appear to be associated with high $L p(a)$ levels were able to be identified without the use of a large number of microarrays.

\section{Expression profiling facilitated discovery of new genes in lipid metabolism}

One of the more common applications of microarray data is for the identification of genes involved in a specifically defined biological process. This annotation of uncharacterized genes is an important process in furthering our cataloging of the functions of the compendium of genes in the human genome. An increasing number of studies have shown that useful insights can be 
made concerning the functions of novel genes based upon the conditions where their expression is altered.

An early example of the usefulness of using microarrays in the identification of disease gene candidates was in the study of Tangiers disease. Among the several near simultaneous reports of the identification of $A B C A 1(A B C 1)$ as the cause of Tangiers disease [8-10] was one which identified the Tangiers gene by the expression profiling of Tangiers disease fibroblasts compared with normal fibroblasts. This analysis identified 175 genes down-regulated more than 2.5 fold (out of a possible 58,800 redundant set) in the Tangiers fibroblasts [11]. Using a combination of data from a second Tangiers patient and genetic mapping information for the disease, $A B C A 1$ was selected for further characterization. Since the initial reports of $A B C A 1$ as the cause of Tangiers disease several other studies have shown the ABCA1 transporter to mediate the efflux of cholesterol from cells $\left[12,13^{*}\right]$. Consistent with this role as an efflux mediator, microarray transcriptional profiling of ABCA1 knockout mouse livers also demonstrated that cholesterol biosynthetic enzymes were decreased compared with wild-type littermates [14*].

Discovery of the genetic basis of the disorder sitosterolemia represents another example where a gene involved in sterol transport was identified through the combination of microarray and genetic map information. Sitosterolemic patients have defects in plant sterol and cholesterol absorption by the intestine and to understand the mechanism of how sterol absorption is controlled Berge et al $\left[15^{\star *}\right]$ used microarrays to search for transcripts that were induced by a liver-X-receptor (LXR) agonist in mouse liver and intestine. Mice treated with an LXR agonist showed a marked decrease in cholesterol absorption suggesting genes involved in this process may be affected. A transcript ABCG5 that showed similarity to the Drosophila ABC halftransporters (brown, scarlet, and white) was induced 2.5-fold in the livers and intestines of treated mice. Since the white gene in humans (ABCG1) has been implicated in cholesterol efflux from macrophages $[16,17]$ it was suspected that ABCG5 was also possibly involved in sterol transport processes. Further evidence for the involvement of ABCG5 in sitosterolemia came from mapping its cDNA to chromosome $2 \mathrm{p} 21$ where the sitosterolemia gene had previously been mapped. Mutations within ABCG5, and the nearby ABCG8 gene, were subsequently shown to be 
responsible for the increased absorption of plant sterols in sitosterolemic patients. This was soon followed by a second study where ABCG5 was implicated in sitosterolemia based purely on mapping data [18].

The use of gene expression patterns to identify the functions of novel genes involved in the metabolism of lipids was further highlighted in a study of 300 expression profiles from yeast that were mutated or chemically treated $\left[19^{\star *}\right]$. In this approach, gene functions were inferred from profile comparisons of transcriptional responses. The identification of Erg 28 as a member of the ergosterol synthesis pathway (the major membrane sterol of yeast) arose out of the analysis because the transcription pattern of Erg 28 closely followed that of other genes involved in sterol synthesis. A human homologue was subsequently identified [20] that was found to be expressed in the testis and some cancer cell lines. By combining the expression patterns of characterized genes that cluster together in a similar transcriptional profile with an uncharacterized gene across many experiments, the investigators were able to associate this gene with a putative biological pathway.

\section{Transcription factors}

Microarrays have found application in the identification of target genes and pathways for many transcription factors $\left[21^{*}\right]$ including nuclear hormone receptors that have major effects upon lipid metabolism. The nuclear hormone receptors are ligand-dependent transcription factors and the biological impacts of nuclear hormone receptor agonists have been examined in several studies [22-25*]. In these studies, the agonists provide a useful way to examine the gene targets of the transcription factors by direct activation. However, other treatments such as hypoxia [26] or oxidized LDL $\left[23^{*}\right]$ have also been demonstrated to activate nuclear hormone receptors. In situations such as this, in which a transcriptional pathway may be affected by a diverse range of conditions, combining the data from all the conditions can help identify those target genes specific to the transcription factor pathway and not due to other effects of the treatment condition.

One group of well studied receptors, the peroxisome proliferator activated receptors (PPARs) regulate expression of enzymes involved in fatty acid oxidation, lipoprotein metabolism, 
adipocyte differentiation and glucose metabolism. In PPARalpha knockout mice, disruption of the PPAR alpha gene resulted in up-regulation of genes associated with amino acid metabolism and the synthesis of amino acid-derived products $\left[27^{*}\right]$. A novel conclusion from this analysis was that the suppressive effect of fatty acids on urea cycle enzymes may be directed through PPAR alpha on ureagenesis and ammonia detoxification. In this case the application of a genome-wide expression study lacking any a priori assumptions led to the identification of new pathways that may be affected by a major transcriptional regulator of fatty acid metabolism.

The involvement of nuclear hormone receptors in atherogenesis and inflammation has also become increasing evident through the use of expression profiling of macrophage foam cells that are intermediary in the development of atherosclerotic lesions [28*]. Related to this, Kohro et al $\left[29^{*}\right]$ found that LXR-alpha was one of the most induced genes in an analysis of macrophage differentiation. This study not only highlighted a new tissue of expression for this important transcriptional regulator, but associated its expression to a cell type implicated in atherosclerotic foam cell formation.

\section{Sequence comparison}

The increasing availability of sequence from multiple organisms has given biologists an opportunity to utilize sequence comparison along with expression profiling to study gene regulation. The rationale for using interspecies sequence comparisons to identify non-coding regulatory elements is based upon the observation that sequences that perform important functions, be they non-coding gene regulatory sequences or important structural domains of a protein, are frequently conserved between species. A crucial component required for the analysis

of genomic sequence to derive biological insights is the availability of "biologist friendly" software. The software needs to be easy to use, configurable, and able to analyze many megabases of sequence from multiple organisms. One of the most popular software programs for comparative sequence studies is VISTA (visualization tool for alignment) which combines a global alignment program with a graphical tool for analyzing alignments $\left[30^{*}, 31^{*}\right]$. An example of how effective the availability of orthologous sequence from multiple species, coupled with visualization tools for 
capturing the information content of cross-species comparisons, is in the identification of noncoding regulatory elements in the constitutive liver enhancer of apo AI (fig 1). The region of the enhancer, which was initially identified from intensive biological studies [32], stands out in a cross species sequence comparison plot (VISTA) as an island of non-coding sequence conservation among non-conserved, non-coding sequences. Similarly, the exons of apo Al also stand out as islands of conservation. While conservation does not tell us what sequence does, its presence provides a genomically informed means to prioritize otherwise anonymous sequences for further biological characterization. The identification of conserved non-coding sequences playing a crucial role in cytokine expression of human chomosome $5 q 31\left[33^{\star}\right]$ is an example of where a conservation of sequence alone lead to the identification of a non-coding region which plays a vital role in the regulation of several genes of importance in the inflammation response.

One of the opportunities as well as challenges in deciphering gene regulation is to identify biologically active transcription factor binding sites. The small number of bases recognized by such mediators of gene expression results in an enormous number of false positive sites in genomic sequence. Advancements in VISTA now allow for the identification of putative transcription factor binding sites based on cross-species conservation which dramatically reduces the number of these sites that are false positives. Regulatory Sequence Analyzer RegSeq (http://www-gsd.lbl.gov/ vista/RegSeq.html) is a computational tool designed for highthroughput discovery of cis-regulatory elements (Loots GG et al, unpublished data). It uses cross-species sequence analysis and clustering of transcription factor binding sites to enrich for evolutionarily conserved sequences. RegSeq maximizes the identification of functional sites and minimizes the number of false-positive transcription factor binding sites, facilitating the discovery of putative transcriptional regulatory elements.

The characterization of important coding as well as non-coding sequences in ABCA1 has been guided by sequence comparison of over $200 \mathrm{~kb}$ surrounding the gene in human and mice [34*]. ABCA1 human-mouse sequence comparison has shown that the conserved promoter region of $A B C A 1$ contains important sequences for the regulation of expression by LXRalpha. Another site of high sequence conservation was found within intron 37 where a conserved non- 
coding sequence of $88 \%$ identity and 498 basepairs was identified. The conserved region was also found in other species including rat, dog, rabbit, bovine, and porcine with 79-88\% homology, however its function remains uncertain.

\section{Discovery of apo AV through comparative sequencing}

Cross-species sequence comparisons used for the discovery of regulatory sequence also can be used as a gene finding aid. This approach recently lead to the discovery of a new apolipoprotein gene in a previously well studied region of the human genome $\left[35^{\star *}\right]$. A human/mouse/rabbit sequence comparison analysis was undertaken of 200 kilobase pairs of the apolipoprotein AI/CIII/AIV region to identify evolutionarily conserved sequences with potential function near this cluster. As observed with many other genes the exons for apo AI, CIII and AIV as well as defined regulatory elements including the apo Al liver specific enhancer, were conserved between human and mouse (fig 2), indicating functional constraints were present in the sequence. However, another large block of sequence conservation was evident $30 \mathrm{~kb}$ upstream of the apo AIV gene. This region demonstrated not just sequence conservation between human and mouse but also sequence similarity to apo AIV at both the DNA level and the predicted protein level. The identification of expressed sequence tags (ESTs) matching the mouse genomic sequence suggested the region was transcribed and analysis of the human and mouse genomic sequence predicted a protein of 366 amino acids and 368 amino acids in these two organisms, respectively. Structural analysis of both the human and mouse proteins predicted the characteristic amphipathic helices found in lipid binding apolipoproteins and antibodies raised to synthetic peptides of apo AV demonstrated the presence of the protein in the VLDL and HDL fractions of plasma of humans and mice providing further evidence that this was a novel apolipoprotein. To assess the function of the protein, transgenic and knockout mice were created and profound, but opposite effects, were seen upon plasma triglyceride levels but not plasma cholesterol levels. Increased levels of human apo AV in transgenic mice lowered triglyceride levels to a third those of normal mice and apo AV deficiency in the knockout mice raised triglyceride levels four-fold. An analysis of human apo AV polymorphisms and lipid parameters in 
two large human association studies with different populations and study designs demonstrated a consistent association between sets of single nucleotide polymorphisms (haplotypes) and triglyceride levels and VLDL mass. The apo AV genomic interval therefore represents a new, independent determinant of triglyceride levels in the human population.

\section{Access to Genomic Resources}

Expression profiling and sequence comparison are two approaches that have been applied with success in the study of gene regulation and gene discovery. Although these two approaches rely heavily upon the availability of genomic resources, the structure of some aspects of biomedical research (following the human genome program example) has changed in that individual research labs are frequently not obligated to generate the genomic data sets for their own research. The broader research community can now access these genomic tools and reagents through a number of programs. One of the most relevant programs for lipid researchers is Programs for Genomic Applications (PGAs) (see table 1) which was established to link genes to biological function and provide a resource for investigators. All of the resources and reagents, including animal models, expression profiles and sequence comparisons being generated by this program are being made available to researchers to advance functional genomic research.

\section{Conclusions}

Many of the successful studies that have utilized expression profiling have also incorporated parallel knowledge about other metabolic or genetic features. In this regard, even very well studied areas have benefited from the application of new technologies. This was most evident in the application of inter-species sequence comparison of the apo Al/CIII/AIV gene cluster. Advancements in how we handle expression data and sequence data will enable us not just to define the individual elements of the genome but to organize them into the complex interaction of elements that regulate gene expression.

\section{Acknowledgements}

We would like to thank Len Pennacchio for useful discussion and figures. 
This work was supported by

NIH project grant HL63897-02 and NHLBI project grant 5U01 HL66681-02. 
Fig 1

A VISTA plot of the apo Al region of human compared with various species. The VISTA (visualization tool for alignment) combines a global alignment program with a graphical tool for analyzing alignments that allows the identification of conserved coding and non-coding sequences between species. The horizontal axis represents the human genomic sequence and the vertical axis indicates the percentage of identical nucleotides in a preset interval between human and another species across the alignment. In this case the analysis uses a 100-nucleotide window, which slides at 40-nucleotide increments. The 4 exons of apo Al are indicated across the top and appear blue on the plot. Non-coding sequence at greater than $75 \%$ identity is shown in red.

Fig 2

Human and mouse comparative sequence analysis of the APOAI/CIII/AIVIAV gene cluster. Above each panel arrows correspond to known genes and their orientation. Exons are depicted in blue. The VISTA graphical plot displays the level of homology between human and the orthologous mouse sequence. Human sequence is represented on the horizontal axis and the percent similarity with the mouse sequence is plotted on the vertical axis. The VISTA plot of the apo Al/CIII/AIVIAV is available at http://pga.lbl.gov/ 


\section{REFERENCES}

1. Lockhart DJ, Dong H, Byrne MC, et al. Expression monitoring by hybridization to highdensity oligonucleotide arrays. Nat Biotechnol 1996;14(13):1675-80.

2. Schena M, Shalon D, Davis RW, Brown PO. Quantitative monitoring of gene expression patterns with a complementary DNA microarray. Science 1995;270(5235):467-70.

*3. Callow MJ, Dudoit S, Gong EL, et al. Microarray expression profiling identifies genes with altered expression in HDL-deficient mice. Genome Res 2000;10(12):2022-9.

In this study t-statistics were used to identify genes that were statistically different between a control group and knockout or transgenic groups.

4. Altman RB, Raychaudhuri S. Whole-genome expression analysis: challenges beyond clustering. Curr Opin Struct Biol 2001;11(3):340-7.

5. Hess KR, Zhang W, Baggerly KA, et al. Microarrays: handling the deluge of data and extracting reliable information. Trends Biotechnol 2001;19(11):463-8.

6. Quackenbush J. Computational analysis of microarray data. Nat Rev Genet 2001;2(6):418-27.

*7. Buechler C, Ullrich H, Ritter M, et al. Lipoprotein (a) up-regulates the expression of the plasminogen activator inhibitor 2 in human blood monocytes. Blood 2001;97(4):981-6.

An example of how single array experiments can be of value in identifying differentially expressed genes.

8. Rust $\mathrm{S}$, Rosier M, Funke $\mathrm{H}$, et al. Tangier disease is caused by mutations in the gene encoding ATP-binding cassette transporter 1. Nat Genet 1999;22(4):352-5.

9. Bodzioch M, Orso E, Klucken J, et al. The gene encoding ATP-binding cassette transporter 1 is mutated in Tangier disease. Nat Genet 1999;22(4):347-51.

10. Brooks-Wilson A, Marcil M, Clee SM, et al. Mutations in ABC1 in Tangier disease and familial high-density lipoprotein deficiency. Nat Genet 1999;22(4):336-45.

11. Lawn RM, Wade DP, Garvin MR, et al. The Tangier disease gene product ABC1 controls the cellular apolipoprotein-mediated lipid removal pathway. J Clin Invest 1999;104(8):R25-31. 
12. Oram JF, Lawn RM. ABCA1. The gatekeeper for eliminating excess tissue cholesterol. J Lipid Res 2001;42(8):1173-9.

*13. Wang N, Silver DL, Thiele C, Tall AR. ATP-binding cassette transporter A1 (ABCA1)

functions as a cholesterol efflux regulatory protein. J Biol Chem 2001;276(26):23742-7.

ABCA1 is involved in phospholipid efflux and apo Al binding, however its role in cholesterol efflux may be indirect.

*14. McNeish J, Aiello RJ, Guyot D, et al. High density lipoprotein deficiency and foam cell accumulation in mice with targeted disruption of ATP-binding cassette transporter-1. Proc Natl Acad Sci U S A 2000;97(8):4245-50.

This study identified a decrease in cholesterol biosynthetic enzymes in liver of ABCA1 knockout mice consistent with its role as mediator of cholesterol efflux.

**15. Berge KE, Tian H, Graf GA, et al. Accumulation of dietary cholesterol in sitosterolemia caused by mutations in adjacent ABC transporters. Science 2000;290(5497):1771-5.

An excellent example of the combination of genetic map data with microarray data to identify disease related genes.

16. Klucken J, Buchler C, Orso E, et al. ABCG1 (ABC8), the human homolog of the Drosophila white gene, is a regulator of macrophage cholesterol and phospholipid transport. Proc Natl Acad Sci U S A 2000;97(2):817-22.

17. Venkateswaran A, Repa JJ, Lobaccaro JM, et al. Human white/murine ABC8 mRNA levels are highly induced in lipid-loaded macrophages. A transcriptional role for specific oxysterols. J Biol Chem 2000;275(19):14700-7.

18. Lee MH, Lu K, Hazard S, et al. Identification of a gene, ABCG5, important in the regulation of dietary cholesterol absorption. Nat Genet 2001;27(1):79-83.

**19. Hughes TR, Marton MJ, Jones AR, et al. Functional discovery via a compendium of expression profiles. Cell 2000;102(1):109-26.

A good example of how co-regulation of genes in a pathway can be valuable in identifying new members of the same pathway. 
20. Gachotte D, Eckstein J, Barbuch R, et al. A novel gene conserved from yeast to humans is involved in sterol biosynthesis. J Lipid Res 2001;42(1):150-4.

*21. Janowski BA, Shan B, Russell DW. The hypocholesterolemic agent LY295427 reverses suppression of SREBP processing mediated by oxysterols. J Biol Chem 2001; 276(48):4540816..

LY295427 reversed the oxysterol-mediated suppression of SREBP processing and subsequently activated multiple SREBP-responsive genes, including the LDL receptor gene which increased metabolism of LDL.

22. Gupta RA, Brockman JA, Sarraf P, et al. Target genes of peroxisome proliferatoractivated receptor gamma in colorectal cancer cells. J Biol Chem 2001;276(32):29681-7. *23. Mikita T, Porter G, Lawn RM, Shiffman D. Oxidized LDL exposure alters the transcriptional response of macrophages to inflammatory stimulus. J Biol Chem 2001; 276(49):45729-39..

An examination of the effects of oxidised LDL and LPS on gene expression in macrophages and the possible involvement of nuclear hormone receptors.

*24. Kerley JS, Olsen SL, Freemantle SJ, Spinella MJ. Transcriptional activation of the nuclear receptor corepressor RIP140 by retinoic acid: a potential negative-feedback regulatory mechanism. Biochem Biophys Res Commun 2001;285(4):969-75.

Retinoic acid was used to identify direct targets of the retinoic acid receptor on 8635-element cDNA arrays and identified RIP140 as consistently upregulated by the treatment.

*25. Schultz JR, Tu H, Luk A, et al. Role of LXRs in control of lipogenesis. Genes Dev $2000 ; 14(22): 2831-8$.

An example of where microarrays have helped in identifying downstream targets of LXR and potential intermediary transcriptional regulators.

26. Narravula S, Colgan SP. Hypoxia-inducible factor 1-mediated inhibition of peroxisome proliferator-activated receptor alpha expression during hypoxia. J Immunol 2001;166(12):7543-8. *27. Kersten S, Mandard S, Escher P, et al. The peroxisome proliferator-activated receptor alpha regulates amino acid metabolism. Faseb J 2001;15(11):1971-8. 
An example of how new pathways, through the use of expression profiling, can be identified for genes associated with alternative pathways.

*28. Shiffman D, Mikita T, Tai JT, et al. Large scale gene expression analysis of cholesterolloaded macrophages. J Biol Chem 2000;275(48):37324-32.

This study grouped differentially expressed genes identified from oxidised-LDL treated macrophages into 7 clusters of expression patterns.

*29. Kohro T, Nakajima T, Wada Y, et al. Genomic structure and mapping of human orphan receptor LXR alpha: upregulation of LXRa mRNA during monocyte to macrophage differentiation. J Atheroscler Thromb 2000;7(3):145-51.

This study highlighted the expression of LXR alpha in macrophages after induction of differentiation by GM-CSF

*30. Mayor C, Brudno M, Schwartz JR, et al. VISTA : visualizing global DNA sequence alignments of arbitrary length. Bioinformatics 2000;16(11):1046-7.

Presents VISTA as a new tool for performing sequence alignments and the identification of conserved elements.

*31. Dubchak I, Brudno M, Loots GG, et al. Active conservation of noncoding sequences revealed by three-way species comparisons. Genome Res 2000;10(9):1304-6.

This study highlights the value in performing multiple species alignments to identify conserved sequences across many species.

32. Walsh A, Ito Y, Breslow JL. High levels of human apolipoprotein A-I in transgenic mice result in increased plasma levels of small high density lipoprotein (HDL) particles comparable to human HDL3. J Biol Chem 1989;264(11):6488-94.

*33. Loots GG, Locksley RM, Blankespoor CM, et al. Identification of a coordinate regulator of interleukins 4,13 , and 5 by cross-species sequence comparisons. Science 2000;288(5463):13640.

This study demonstrates the removal of conserved sequences from a transgene and the impact upon the expression of T-cell interleukins 
*34. Qiu Y, Cavelier L, Chiu S, et al. Human and mouse ABCA1 comparative sequencing and transgenesis studies revealing novel regulatory sequences. Genomics 2001;73(1):66-76.

Comparative sequence alignment identified potentially important sequences in an intron of the ABCA1 gene.

**35. Pennacchio LA, Olivier M, Hubacek JA, et al. An apolipoprotein influencing triglycerides in humans and mice revealed by comparative sequencing. Science 2001;294(5540):169-73.

This study encompasses characterization of knockout and transgenic mice and two independent SNP association studies to clearly implicate apo AV as a determining factor in triglyceride metabolism. 